\title{
Oral vaccine of Lactococcus lactis harbouring pandemic H1N1 2009 haemagglutinin1 and nisP anchor fusion protein elevates anti-HA1 sIgA levels in mice
}

\begin{abstract}
Objective: An oral lactococcal-based vaccine which haboured the haemagglutinin1 (HA1) antigen fused to nisP anchor protein for the purpose of surface displaying the HA1 antigen was developed against H1N1 virus. Results: Recombinant L. lactis strains expressed HA1nisP fusion proteins when induced with nisin, as confirmed through western blotting. However, immunofluorescense did not detect any surface-displayed proteins, suggesting that the protein was either unsuccessfully translocated or improperly displayed. Despite this, oral administration of recombinant $\mathrm{L}$. lactis strains to $\mathrm{BALB} / \mathrm{c}$ mice revealed that significant levels of anti-HA1 sIgA antibodies were detected in mice fecal suspension samples of mice group NZ9000 (pNZ:HN) when compared to the negative control NZ9000 (pNZ8048) group. Conclusion: Specific anti-HA1 sIgA antibodies were locally produced and live recombinant lactococcal vaccine was able to elicit humoral response of BALB/c mice despite unsuccessful surface display of the HA1 epitope.
\end{abstract}

Keyword: H1N1; Haemagglutinin; Influenza virus; Lactococcus lactis; Oral vaccine; Surface display; Vaccine 